\title{
Qualidade de vida de puérperas que vivenciaram near miss materno
}

\author{
Quality of life of puerperal women who experienced maternal near miss
}

Calidad de vida de las puérperas que experimentaron materno cuasi accidentes

Natália Carolina Rodrigues Colombo ${ }^{1 *}$, Cristiane de Melo Aggio $^{2}$, Renne Rodrigues ${ }^{3}$ Fabiana Fontana Medeiros ${ }^{4}$, Lailla Ketly Ferreira Tiradentes Ruiz ${ }^{5}$, Franciane Maria da Silva Curan ${ }^{6}$, Izabel Dayana de Lemos Santos ${ }^{7}$, Alexandrina Aparecida Maciel Cardelli ${ }^{8}$.

\section{RESUMO}

Objetivo: Avaliar a qualidade de vida de mulheres que vivenciaram o near miss no período pós-parto. Métodos: Estudo transversal, aninhado a uma coorte prospectiva, entre outubro de 2016 e agosto de 2017. Participaram 262 puérperas de alto risco de uma maternidade pública, sem comprometimento cognitivo, com algum critério de near miss materno, não submetidas ao abortamento ou tratamento gestacional. Utilizou-se formulário estruturado para a caracterização delas e o Short Form Survey, cujas medianas e testes de MannWhitney, Kruskal-Wallis e de Dunn foram calculados. Resultados: Dor e limitações por aspectos físicos e emocionais foram os domínios da qualidade de vida com piores escores e o near miss cardiovascular prevaleceu. Adolescentes tiveram pior escore de limitação física $(p=0,035)$, puérperas com 36 anos ou mais apresentaram melhor escore de saúde mental $(p=0,042)$, as brancas apresentaram escore pior de dor ( $p$ $=0,04)$ e melhor de estado geral de saúde $(p=0,04)$ e quem realizou cesárea teve pior escore de limitação física, aspectos emocionais, saúde mental e aspectos globais físicos e mentais. Conclusão: A qualidade de vida das participantes foi prejudicada pela dor e limitações físicas e emocionais, que definem o diagnóstico de conforto prejudicado e exigem qualidade na atenção à saúde materna.

Palavras-chave: Qualidade de vida, Saúde materna, Near miss.

\section{ABSTRACT}

Objective: To assess the quality of life of women who experienced near miss in the postpartum period. Methods: Cross-sectional study, nested in a prospective cohort, between October 2016 and August 2017. 262 high-risk postpartum women in a public maternity, without cognitive impairment, with some maternal near miss criterion, not undergoing abortion or gestational treatment, participated. A structured form was used to characterize them and the Short Form Survey, whose medians and Mann-Whitney, Kruskal-Wallis and Dunn tests were calculated. Results: Pain and limitations due to physical and emotional aspects were the domains of quality of life with the worst scores and cardiovascular near miss prevailed. Adolescents had worse physical limitation score $(p=0.035)$, puerperal women aged 36 years or more had better mental health score $(p=$ $0.042)$, white women had worse pain score $(p=0.04)$ and better general condition health $(p=0.04)$ and those who underwent cesarean section had a worse score for physical limitations, emotional aspects, mental health and overall physical and mental aspects. Conclusion: The participants' quality of life was impaired by pain and physical and emotional limitations, which define the diagnosis of impaired comfort and demand quality in maternal health care.

Keywords: Quality of life, Maternal health, Near miss.

${ }^{1}$ Universidade Estadual de Londrina (UEL), Londrina - PR.

2 Universidade Estadual do Centro Oeste (UNICENTRO), Guarapuava - PR. *E-mail: colombo@uel.br 


\section{RESUMEN}

Objetivo: Evaluar la calidad de vida de las mujeres que experimentaron casi accidentes en el período posparto. Métodos: Estudio transversal, anidado en una cohorte prospectiva, entre octubre de 2016 y agosto de 2017. Participaron 262 mujeres posparto de alto riesgo en una maternidad pública, sin deterioro cognitivo, con algún criterio de cuasi maternidad, no sometidas a aborto ni tratamiento gestacional. Para caracterizarlos se utilizó una forma estructurada y la Encuesta de Forma Corta, cuyas medianas y pruebas de Mann-Whitney, Kruskal-Wallis y Dunn se calcularon. Resultados: el dolor y las limitaciones por aspectos físicos y emocionales fueron los dominios de calidad de vida con peores puntuaciones y predominó el near miss cardiovascular. Las adolescentes tuvieron peor puntuación de limitación física $(p=0,035)$, las puérperas de 36 años o más tuvieron una mejor puntuación de salud mental $(p=0,042)$, las mujeres blancas tuvieron peor puntuación de dolor $(p=0,04)$ y mejor estado general salud $(p=0,04)$ y las que se sometieron a cesárea tuvieron una peor puntuación en las limitaciones físicas, los aspectos emocionales, la salud mental y los aspectos físicos y mentales en general. Conclusión: La calidad de vida de las participantes se vio afectada por el dolor y las limitaciones físicas y emocionales, que definen el diagnóstico de deterioro de la comodidad y calidad de la demanda en la atención de la salud materna.

Palabras clave: Calidad de vida, Salud materna, Near miss.

\section{INTRODUÇÃO}

A qualidade de vida diz respeito à percepção da pessoa sobre a sua posição na vida e na sociedade na qual está inserido, incluindo suas metas, expectativas, padrões e preocupações. Este conceito abrange os aspectos físicos e psicológicos da saúde, a independência, crenças, relações sociais e fatores ambientais (OMS, 2011).

Nesta perspectiva, a saúde deixa de ser definida pela inexistência de doenças e passa a considerar o amplo e complexo estado de completo bem-estar físico, mental e social, afetado por diversos fatores, como as condições de trabalho, questões de gênero, estilo de vida, exclusão social, violência e violação dos direitos humanos (WHO, 2020). Mudanças nestes domínios podem determinar alterações no estado de saúde e refletir na qualidade de vida do indivíduo (CONDELES PC, et al., 2019). Na saúde materna destaca-se o período pós-parto, também chamado de puerpério, enquanto um momento que agrega inúmeras transformações decorrentes de outras demandas para mulher, caracterizadas por novas responsabilidades e um novo modo de estar no mundo (REZENDE FILHO J e MONTENEGRO CAB, 2014).

O período pós-parto compreende uma fase delicada, cheia de adaptações e evolução única para cada mulher, na qual ela necessita se adequar às modificações biológicas, exercício da maternidade, cuidados com o filho, novo contexto familiar, demandas psicológicas, relacionais, socioculturais, econômicas, além das questões de gênero, as quais são vivenciadas conforme os valores, crenças e costumes das mesmas (CABRAL FB e LIVEIRA DLLC, 2010).

Outro componente importante a ser considerado no período puerperal é a falta de suporte familiar e social nesse momento, podendo gerar desgastes físicos, distúrbios de humor, estresse e sintomas de depressão nessas mulheres (OKUN ML, et al., 2018). Além de todas essas transformações vivenciadas no puerpério, há uma parcela de mulheres que por conviverem com alguma doença ou sofrerem algum agravo apresentam maiores chances de um desfecho desfavorável tanto para a mãe como para seu filho (OMS, 2011).

Um dos indicadores da saúde materna é a razão de mortalidade materna (RMM), que é constituída do número de mortes maternas durante um ano por 100 mil nascidos vivos durante o mesmo período e reflete o risco obstétrico associado à gestação, sendo recomendados valores inferiores à 20 mortes por 100.000 Nascidos Vivos (NV) (WHO, 2020).Por também representar a qualidade de atenção à saúde da mulher, as elevadas taxas deste indicador nos levam a questionar como vem sendo prestados os serviços de saúde, desde o planejamento familiar, assistência pré-natal, parto e puerpério, haja visto que, apesar do declínio evidenciado após a implantação da Rede Mãe Paranaense de 2012, a RMM em 2017 foi de 20,3\% (PARANÁ, 2018). 
Para tentar reduzir a RMM, os serviços de saúde devem identificar, avaliar e acompanhar as mulheres que sobreviveram às complicações graves da gravidez, parto e pós-parto, as quais têm muitos aspectos similares aos das que morreram por esta causa, caracterizando os casos de near miss materno (NMM), mulheres que sofreram algum evento de "quase morte" mas sobreviveram, e indicarão os problemas e obstáculos da atenção à saúde materna (DIAS MAB, et al., 2014; LONGHI SAT, PETERLINI OLG, 2019).

Conhecer as dimensões comprometidas da qualidade de vida das puérperas vítimas de NMM favorecerá a formulação e implementação de ações de promoção, proteção e recuperação da saúde no período pósparto. Assim, este estudo teve como objetivo avaliar a qualidade de vida de mulheres que vivenciaram o near miss no período pós-parto.

\section{MÉTODOS}

Trata-se de um estudo transversal, aninhado à uma coorte prospectiva, o qual foi realizado em uma maternidade pública do sul do Brasil, que é uma referência do SUS para gestantes de alto risco. Ela abrange

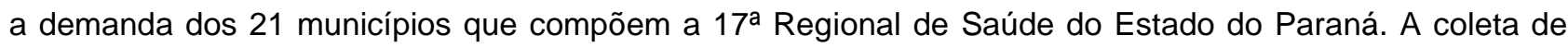
dados ocorreu no período de outubro de 2016 a agosto de 2017, por meio de entrevistas estruturadas. Foram incluídas as puérperas internadas na maternidade em questão, sem comprometimento cognitivo e que apresentavam ao menos um dos critérios de NMM, apresentados no Quadro 1. Foram excluídas aquelas que realizaram abortamento ou tratamento gestacional.

Quadro 1 - Critérios de near miss materno estabelecidos pela OMS.

\begin{tabular}{|c|c|}
\hline $\begin{array}{l}\text { Complicações maternas graves: } \\
\text { - Hemorragia pós-parto grave } \\
\text { - Pré-eclâmpsia grave } \\
\text { - Eclâmpsia } \\
\text { - Sepse ou infecção sistêmica grave } \\
\text { - Rotura uterina } \\
\text { • Complicações graves do abortamento. }\end{array}$ & $\begin{array}{l}\text { Intervenções críticas ou uso da unidade de } \\
\text { terapia intensiva: } \\
\text {-Internação em uma unidade de terapia intensiva } \\
\text { - Radiologia intervencionista } \\
\text { - Laparotomia (inclui histerectomia, exclui cesáreas) } \\
\text { - Uso de hemoderivados. }\end{array}$ \\
\hline $\begin{array}{l}\text { Condições ameaçadoras à vida (critérios de } \\
\text { near miss) } \\
\text { - Disfunção cardiovascular: choque, parada } \\
\text { cardíaca, uso contínuo de drogas vasoativas, } \\
\text { reanimação cardiopulmonar, hipoperfusão grave } \\
\text { (lactato >5 mmol// ou >45 mg/dl), acidose grave } \\
\text { (pH <7,1); } \\
\text { - Disfunção respiratória: cianose aguda, } \\
\text { respiração tipo Gasping, taquipneia grave } \\
\text { (frequência respiratória >40 respirações/minuto), } \\
\text { bradipneia grave (frequência respiratória <6 } \\
\text { respirações/minuto), intubação e ventilação não } \\
\text { relacionadas à anestesia, hipoxemia grave } \\
\text { (saturação de oxigênio <90\% por tempo } \geq 60 \\
\text { minutos ou PaO2/ FiO2 <200). }\end{array}$ & $\begin{array}{l}\text {-Disfunção renal: oligúria não responsiva aos } \\
\text { fluidos ou diuréticos, diálise para insuficiência renal } \\
\text { aguda, azotemia aguda grave (creatinina } \geq 300 \\
\mu \mathrm{mol} / \mathrm{ml} \text { ou } \geq 3,5 \mathrm{mg} / \mathrm{dl} \text { ); } \\
\text {-Disfunção hematológica da coagulação: falência } \\
\text { da coagulação, grande transfusão de sangue ou de } \\
\text { hemácias ( } \geq 5 \text { unidades), trombocitopenia aguda } \\
\text { grave ( }<50.000 \text { plaquetas/ml); } \\
\text { - Disfunção hepática: icterícia na presença de pré- } \\
\text { eclâmpsia, hiperbilirrubinemia (bilirrubina }>100 \mu \text { mol/l } \\
\text { ou >6,0 mg/dl); } \\
\text { - Disfunção neurológica: perda de consciência } \\
\text { prolongada (duração } \geq 12 \text { horas)/coma (incluindo o } \\
\text { metabólico), acidente vascular cerebral, convulsões } \\
\text { incontroláveis/status epilético, paralisia total; } \\
\text { - Disfunção uterina: hemorragia ou infecção uterina } \\
\text { causadores de histerectomia; }\end{array}$ \\
\hline $\begin{array}{l}\text { Status vital materno } \\
\text { - Óbito materno. }\end{array}$ & \\
\hline
\end{tabular}

Fonte: Colombo NCR, et al., 2020. Baseado em OMS, 2011. 
Considerou-se o cálculo amostral estimado de 262 puérperas e utilizou-se um formulário estruturado para caracterização da população, que possuía 340 questões relacionadas à história obstétrica atual, do cartão de pré-natal e prontuário hospitalar, bem como o Short Form Survey (SF-36), que avalia oito domínios da qualidade de vida relacionada à saúde (QVRS), em escores de zero a 100, sendo o zero o pior escore de qualidade de vida da pessoa naquele domínio (CICONELLI RM, 1997).

Os dados coletados foram tratados no programa Statistical Package for the Social Sciences ${ }^{\circledR}$ (SPSS), versão 20.0. Para caracterização das mulheres foi realizada a análise exploratória, sendo os dados apresentados em mediana (mínimo - máximo ou 95\% IC) ou frequência absoluta (n) e frequência relativa (\%). Para verificar a homogeneidade dos dados, foram utilizados os testes Shapiro-Wilk e o de Levene, conforme necessário. A comparação das medianas foi realizada utilizando o teste de Mann-Whitney ou Kruskal-Wallis. Quando necessário, o teste de post-hoc de Dunn foi utilizado. Para todas as comparações foi adotado o nível de significância de $p<0,05$. Esta pesquisa foi aprovada pelo Comitê de Ética em Pesquisa Envolvendo Seres Humanos da Universidade Estadual de Londrina (CEP/UEL), parecer 1.757.596.

\section{RESULTADOS}

Sobre as características sociodemográficas das puérperas em situação de NMM destacaram-se as mulheres com idade entre 20 e 35 anos $(70,6 \%)$, as que se auto declararam brancas $(58,6 \%)$, as que tinham companheiro $(88,2 \%)$, as que estudaram até o ensino médio $(83,9 \%)$, as que possuíam renda de até dois salários mínimos $(50,7 \%)$ e aquelas que tinham ocupações com nível de atividade física leve (77, 5\%). A cesariana foi o principal tipo de parto das puérperas em situação de NMM $(61,1 \%)$ e a maioria delas era primípara $(40,5 \%)$, classificou a atenção ao pré-natal como adequado $(99,2 \%)$ e tiveram seis ou mais consultas de pré-natal $(92,2 \%)$, as quais foram eficientes para $72,9 \%$ das participantes.

Entre os oito domínios da qualidade de vida das puérperas em situação de NMM, a dor (mediana: 21,0), as limitações por aspectos físicos (mediana: 25,0) e as limitações por aspectos emocionais (mediana: 33,3 ) foram os únicos que apresentaram a mediana dos escores inferior a 50 , sendo que os valores atribuídos a cada um destes domínios variaram entre zero e 100. Na comparação entre os domínios da qualidade de vida e as características sociodemográficas, condição econômica, tipo de parto e número de filhos não foram encontradas diferenças significativas para os desfechos situação conjugal, escolaridade, renda, classificação da ocupação e número de filhos ( $p>0,05)$.

Em relação a faixa etária, as mulheres mais jovens (14-19 anos), apresentaram pior percepção de limitação física em relação as mulheres com idade superior ou igual a 36 anos ( $p=0,035)$. Por outro lado, as mulheres com 36 anos ou mais tiveram melhor percepção de saúde mental em relação às mais jovens $(p=0,042)$. Quanto à raça, as mulheres brancas apresentaram piores escores para dor e melhores escores para o estado geral de saúde em comparação com as mulheres negras ( $p=0,04$ para ambos).

Por fim, as mulheres que realizaram cesárea, apresentaram piores escores de qualidade de vida nos domínios limitação física, aspectos emocionais, saúde mental e aspectos globais físicos e mentais quando comparadas àquelas que realizaram parto normal espontâneo (Tabela 1).

Os eventos de NMM de origem cardiovascular (hipertensão, pré-eclâmpsia e cardiopatia) foram os mais prevalentes na amostra estudada $(54,6 \%)$, seguido dos metabólicos $(39,3 \%)$ (obesidade, diabetes mellitus e distúrbios da tireoide), infecciosos (14,1\%) (ITU e infeccioso) e hematológicos (9,2\%) (distúrbios de coagulação e hemorragia).

Foram encontradas diferenças significativas nos domínios de limitação física e aspectos sociais apenas entre as pacientes com eventos de NMM de origem metabólica. Neste contexto, as mulheres com NMM metabólico, apresentaram pior qualidade de vida para a limitação física $(p=0,047)$ e para os aspectos sociais $(p=0,020)$, em comparação com as mulheres que não desenvolveram NMM de origem metabólica. Não foram identificadas outras diferenças significativas $(p>0,05)$ para todas as outras comparações (Tabela 2). Não foram identificadas outras diferenças significativas $(p>0,05)$ para todas as outras comparações. 
Tabela 1 - Comparação dos domínios de qualidade de vida de acordo com a caracterização sociodemográfica, econômica, tipo de parto e número de filhos, de puérperas em situação de NMM durante a internação, 17ª RS, Paraná, 2020 (Dados apresentados em mediana e IC 95\%).

\begin{tabular}{|c|c|c|c|c|c|c|c|c|c|c|}
\hline $\begin{array}{c}\text { Características } \\
\text { sociodemográficas }\end{array}$ & $\begin{array}{l}\text { Capacidade } \\
\text { Funcional }\end{array}$ & $\begin{array}{l}\text { Limitação } \\
\text { Física }\end{array}$ & Dor & $\begin{array}{c}\text { Estado } \\
\text { Geral de } \\
\text { Saúde }\end{array}$ & Vitalidade & $\begin{array}{l}\text { Aspectos } \\
\text { sociais }\end{array}$ & $\begin{array}{l}\text { Aspectos } \\
\text { Emocionais }\end{array}$ & $\begin{array}{l}\text { Saúde } \\
\text { Mental }\end{array}$ & $\begin{array}{l}\text { Aspectos } \\
\text { Físicos } \\
\text { Global }\end{array}$ & $\begin{array}{c}\text { Aspectos } \\
\text { Mentais } \\
\text { Global }\end{array}$ \\
\hline \multicolumn{11}{|l|}{ Faixa etária } \\
\hline $14-19$ & $\begin{array}{c}85,0 \\
(62,0-81,5)\end{array}$ & $\begin{array}{c}50,0 \\
(36,5- \\
67,0)\end{array}$ & $\begin{array}{c}32,0 \\
(23,8- \\
37,0)\end{array}$ & $\begin{array}{c}65,0 \\
(58,1- \\
67,5)\end{array}$ & $\begin{array}{c}55,0 \\
(49,0- \\
67,0)\end{array}$ & $\begin{array}{c}75,0 \\
(66,0- \\
80,4)\end{array}$ & $\begin{array}{c}67,0 \\
(37,8-72,6)\end{array}$ & $\begin{array}{c}80,0 \\
(67,6- \\
82,0)\end{array}$ & $\begin{array}{c}55,5 \\
(48,0- \\
60,4)\end{array}$ & $\begin{array}{c}66,2 \\
(57,7- \\
72,6)\end{array}$ \\
\hline $20-35$ & $\begin{array}{c}85,0 \\
(73,1-79,7)\end{array}$ & $\begin{array}{c}25,0 \\
(37,5- \\
49,2)\end{array}$ & $\begin{array}{c}21,0 \\
(27,2- \\
33,9)\end{array}$ & $\begin{array}{c}60,0 \\
(57,5- \\
62,0)\end{array}$ & $\begin{array}{c}50,0 \\
(50,3- \\
56,5)\end{array}$ & $\begin{array}{c}63,0 \\
(64,6- \\
71,5)\end{array}$ & $\begin{array}{c}33,0 \\
(41,3-53,4)\end{array}$ & $\begin{array}{c}68,0 \\
(63,5- \\
69,2)\end{array}$ & $\begin{array}{c}50,2 \\
(50,3- \\
55,0)\end{array}$ & $\begin{array}{c}57,8 \\
(56,0- \\
61,6)\end{array}$ \\
\hline$\geq 36$ & $\begin{array}{c}80,0 \\
(63,3-79,6)\end{array}$ & $\begin{array}{c}80,0 \\
(18,1- \\
41,3)\end{array}$ & $\begin{array}{c}21,0 \\
(24,0- \\
38,0)\end{array}$ & $\begin{array}{c}55,0 \\
(54,6- \\
63,5)\end{array}$ & $\begin{array}{c}50,0 \\
(49,0- \\
59,3)\end{array}$ & $\begin{array}{c}63,0 \\
(59,7- \\
73,0)\end{array}$ & $\begin{array}{c}33,0 \\
(32,1-57,0)\end{array}$ & $\begin{array}{c}66,0 \\
(57,9- \\
70,0)\end{array}$ & $\begin{array}{c}45,2 \\
(43,0- \\
53,0)\end{array}$ & $\begin{array}{c}58,3 \\
(51,6- \\
62,6)\end{array}$ \\
\hline $\mathrm{p}$-valor KW & 0,506 & 0,035 & 0,479 & 0,414 & 0,521 & 0,414 & 0,574 & 0,042 & 0,130 & 0,181 \\
\hline \multicolumn{11}{|l|}{ Raça/cor } \\
\hline Branca & $\begin{array}{c}85,0 \\
(72,5-80,1)\end{array}$ & $\begin{array}{c}25,0 \\
(33,2- \\
46,1)\end{array}$ & $\begin{array}{c}21,0 \\
(25,3- \\
32,2)\end{array}$ & $\begin{array}{c}60,0 \\
(58,7- \\
63,4)\end{array}$ & $\begin{array}{c}50,0 \\
(48,5- \\
55,4)\end{array}$ & $\begin{array}{c}63,0 \\
(62,4- \\
70,1)\end{array}$ & $\begin{array}{c}33,0 \\
(40,6-54,3)\end{array}$ & $\begin{array}{c}68,0 \\
(61,5- \\
68,0)\end{array}$ & $\begin{array}{c}48,5 \\
(48,9- \\
54,0)\end{array}$ & $\begin{array}{c}56,9 \\
(54,4- \\
61,0)\end{array}$ \\
\hline Negra & $\begin{array}{c}80,0 \\
(68,1-77,7)\end{array}$ & $\begin{array}{c}37,5 \\
(37,2- \\
53,2)\end{array}$ & $\begin{array}{c}21,0 \\
(28,8- \\
38,3)\end{array}$ & $\begin{array}{c}55,0 \\
(54,9- \\
60,6)\end{array}$ & $\begin{array}{c}55,0 \\
(52,6- \\
60,6)\end{array}$ & $\begin{array}{c}75,0 \\
(66,4- \\
74,9)\end{array}$ & $\begin{array}{c}33,0 \\
(38,8-54,8)\end{array}$ & $\begin{array}{c}72,0 \\
(65,5- \\
73,1)\end{array}$ & $\begin{array}{c}52,5 \\
(49,1- \\
55,6)\end{array}$ & $\begin{array}{c}59,3 \\
(57,4- \\
64,3)\end{array}$ \\
\hline p-valor MW & 0,250 & 0,290 & 0,043 & 0,041 & 0,050 & 0,127 & 0,897 & 0,059 & 0,627 & 0,155 \\
\hline \multicolumn{11}{|l|}{ Tipo de parto } \\
\hline Normal espontâneo & $\begin{array}{c}85,0 \\
(72,8-81,0)\end{array}$ & $\begin{array}{c}50,0 \\
(40,0- \\
56,1)\end{array}$ & $\begin{array}{c}21,0 \\
(27,8- \\
37,1)\end{array}$ & $\begin{array}{c}60,0 \\
(58,3- \\
64,2)\end{array}$ & $\begin{array}{c}55,0 \\
(52,1- \\
61,0)\end{array}$ & $\begin{array}{c}63,0 \\
(65,7- \\
74,6)\end{array}$ & $\begin{array}{c}67,0 \\
(47,2-64,0)\end{array}$ & $\begin{array}{c}76,0 \\
(66,4- \\
74,2)\end{array}$ & $\begin{array}{c}54,1 \\
(51,5- \\
57,7)\end{array}$ & $\begin{array}{c}65,3 \\
(59,2- \\
67,0)\end{array}$ \\
\hline Cesária & $\begin{array}{c}85,0 \\
(69,7-77,9)\end{array}$ & $\begin{array}{c}25,0 \\
(31,6- \\
44,0)\end{array}$ & $\begin{array}{c}21,0 \\
(26,0- \\
33,0)\end{array}$ & $\begin{array}{c}60,0 \\
(57,0- \\
61,4)\end{array}$ & $\begin{array}{c}50,0 \\
(49,3- \\
56,0)\end{array}$ & $\begin{array}{c}63,0 \\
(63,4- \\
70,8)\end{array}$ & $\begin{array}{c}33,0 \\
(36,2-49,1)\end{array}$ & $\begin{array}{c}64,0 \\
(61,5- \\
67,7)\end{array}$ & $\begin{array}{c}47,9 \\
(47,5- \\
52,5)\end{array}$ & $\begin{array}{c}54,7 \\
(54,0- \\
59,6)\end{array}$ \\
\hline $\mathrm{p}$-valor MW & 0,685 & 0,048 & 0,236 & 0,340 & 0,182 & 0,476 & 0,020 & 0,012 & 0,044 & 0,009 \\
\hline
\end{tabular}

Nota: KW= Kruskal-Wallis. MW = Mann-Whitney; ap <0,05 vs. $\geq 36$ anos; Fonte: Colombo NCR, et al., 2020. 
Tabela 2 - Comparação dos domínios de qualidade de vida de acordo com a origem do near miss de puérperas durante a internação, 17ª RS, Paraná, 2020 (Dados apresentados em mediana e 95\% IC).

\begin{tabular}{|c|c|c|c|c|c|c|c|c|c|c|}
\hline Near miss & $\begin{array}{l}\text { Capacidade } \\
\text { Física }\end{array}$ & $\begin{array}{l}\text { Limitação } \\
\text { Física }\end{array}$ & Dor & $\begin{array}{l}\text { Estado } \\
\text { Geral de } \\
\text { Saúde }\end{array}$ & Vitalidade & $\begin{array}{l}\text { Aspectos } \\
\text { Sociais }\end{array}$ & $\begin{array}{l}\text { Aspectos } \\
\text { Emocionais }\end{array}$ & $\begin{array}{l}\text { Saúde } \\
\text { Mental }\end{array}$ & $\begin{array}{l}\text { Aspectos } \\
\text { Físicos } \\
\text { Global }\end{array}$ & $\begin{array}{c}\text { Aspectos } \\
\text { Mentais } \\
\text { Global }\end{array}$ \\
\hline \multicolumn{11}{|l|}{ Metabólico } \\
\hline \multirow{2}{*}{ Sim } & 85,0 & & & 60,0 & 50,0 & 63,0 & 33,0 & 68,0 & 49,0 & 57,3 \\
\hline & $(70,8-80,4)$ & $\begin{array}{c}(28,0- \\
43,3)\end{array}$ & $\begin{array}{c}(24,9- \\
33,2)\end{array}$ & $\begin{array}{c}(56,6- \\
62,6)\end{array}$ & $\begin{array}{c}(49,1- \\
56,9)\end{array}$ & $\begin{array}{c}(59,8- \\
69,1)\end{array}$ & $(38,5-55,3)$ & $\begin{array}{l}(63,4- \\
70,3)\end{array}$ & $\begin{array}{c}(46,8- \\
53,2)\end{array}$ & $\begin{array}{l}(53,9- \\
61,6)\end{array}$ \\
\hline \multirow{2}{*}{ Não } & 80,0 & 50,0 & 21,0 & 60,0 & 55,0 & 75,0 & 33,0 & 68,0 & 51,3 & 59,5 \\
\hline & $(70,9-78,3)$ & $\begin{array}{c}(39,3- \\
52,2)\end{array}$ & $\begin{array}{l}(27,9- \\
35,2)\end{array}$ & $\begin{array}{c}(57,9- \\
62,5)\end{array}$ & $\begin{array}{c}(51,3- \\
58,1)\end{array}$ & $\begin{array}{l}(67,3- \\
74,3)\end{array}$ & $(41,6-54,8)$ & $\begin{array}{l}(63,5- \\
70,1)\end{array}$ & $\begin{array}{c}(50,5- \\
55,5)\end{array}$ & $\begin{array}{l}(57,1- \\
63,1)\end{array}$ \\
\hline $\mathrm{p}$-valor MW & 0,591 & 0,047 & 0,398 & 0,677 & 0,357 & 0,020 & 0,800 & 0,797 & 0,165 & 0,329 \\
\hline
\end{tabular}

Nota: $\mathrm{KW}=$ Kruskal-Wallis. MW = Mann-Whitney

Fonte: Colombo NCR, et al., 2020. 


\section{DISCUSSÃO}

Além do prejuízo na qualidade de vida das puérperas, os casos de NMM no período pós-parto indica a presença de atrasos no processo de cuidar e fragilidades na qualidade da assistência oferecida pela maternidade estudada e o reconhecimento dos mesmos auxiliará a definição de medidas a serem tomadas para que este desfecho seja rapidamente identificado e evitado (SANTANA DS, et al., 2018). As mulheres classificadas como adultas jovens, brancas, que possuem companheiro, baixa escolaridade e renda, primíparas e que realizaram parto cesariano predominaram neste estudo. Tais características foram associadas à morbidade materna e à ocorrência de NMM, em diversos estudos nacionais e internacionais (VIDAL CEL, et al., 2016; PINHEIRO DLFL, et al., 2020; CHICAS AAN, et al., 2018).

Quanto a idade, as adolescentes obtiveram maiores pontuações nos domínios limitação física e saúde mental o que corrobora com um estudo que afirma que os extremos de idade influenciaram negativamente na qualidade de vida (ANGELINI CR, et al., 2018). Outro estudo, porém, identificou influência de características sociodemográficas na qualidade de vida das puérperas afirmando a necessidade de uma rede de apoio com efeito favorável para as adolescentes (SILVA SGF, et al., 2019).

A maior ocorrência de cesáreas pode estar relacionada às complicações no estado clínico das parturientes, com consequências no desempenho de atividades no puerpério e na qualidade de vida relacionada às limitações físicas e emocionais, principalmente no que concerne à dor e a sensação de incapacidade (MEDEIROS TML e MARCELINO JFQ, 2018).

Quando comparadas às puérperas de baixo risco, as que sofreram NMM apresentaram escores mais baixos de qualidade de vida em todos os domínios (SOMA-PILLAY P, et al., 2018), sendo que as complicações intraparto repercutem na saúde global da mulher a longo prazo, principalmente relacionado a aspectos físicos e a experiência com o nascimento do filho, por não terem suas necessidades respeitadas e atendidas (AGUIAR CA e TANAKA ACD, 2016).

Neste estudo um pouco mais que a metade das puérperas apresentaram NMM de origem cardiovascular, assim como as gestantes e puérperas de uma maternidade de Uberlândia-MG, que atendia mulheres com gestação de alto risco, cuja maioria era negra e parda, com idade entre 20 e 39 anos, submetida à cesárea atual, destacando-se o choque, oligúria não responsiva à hidratação e padrão respiratório alterado entre os critérios clínicos apresentados por elas (ARANTES BM, et al., 2020).

As puérperas vítimas de NMM de origem cardiovascular e hemorrágica, assistidas na maternidade de um hospital universitário do Paquistão foram urgentemente submetidas à hemodiálise, infusão de sulfato de magnésio, reembolização da artéria uterina, histerectomia de emergência e ventilação não invasiva e invasiva, com o intuito de se obter resultados favoráveis à saúde materna (SULTANA S, et al., 2019).

Assim como neste estudo, em outro país em desenvolvimento, o NMM por distúrbios cardiovasculares e hematológicos predominaram e tiveram os mais baixos índices de mortalidade materna, possivelmente devido à prevenção e ao manejo adequado da hemorragia obstétrica neste outro país, sugerindo uma qualidade razoável da atenção à mulher no período pós-operatório (IWUH IA, et al., 2018).

A qualidade da atenção à puérpera foi ratificada por enfermeiras de uma maternidade de Manaus-AM, que também é referência para gestantes de alto risco, cuja maioria tinha especialização me ginecologia/obstetrícia, nunca tinha atendido um caso deste e afirmava conhecer as causas da hemorragia no período pós-parto, bem como suas medidas preventivas e de controle, mesmo não havendo um procedimento operacional padrão descrito para o cuidado de enfermagem para esta clientela (VIEIRA SN, et al., 2018).

As patologias metabólicas foram associadas a menores escores de qualidade de vida, o que foi semelhante a outro estudo que mostrou que características clínicas pregressas como doenças respiratórias, distúrbios da tireóide e HIV foi desfavorável à qualidade de vida (ANGELINI CR, et al., 2018). As doenças hematológicas, hipertensivas e cardiovasculares são as mais frequentes nesta população, com ocorrência de $55,3 \%$ no grupo NMM e $33,2 \%$ no controle, além de obesidade, diabetes, tabagismo, doenças renais, neurológicas e respiratórias (MEKANGO DE, et al., 2017; FERREIRA EC, et al., 2020). 
A relação do distúrbio hematológico com a qualidade de vida não possui explicação na literatura, no entanto, pode-se inferir que a permanência das patologias hematológicas no pós-parto influencie a percepção da mulher quanto a sua saúde geral. Neste estudo a dor e as limitações físicas e emocionais foram as dimensões da qualidade de vida das puérperas mais afetadas após a vivência do NMM, as quais foram confirmadas pelos valores globais da qualidade de vida e dizem respeito ao diagnóstico de enfermagem de conforto prejudicado (FIGUEIREDO JV, et al., 2018).

Estes achados se assemelham aos das puérperas da Holanda, Canadá e Suécia que apresentavam doenças na gestação e/ou puerpério. Possivelmente estas dimensões prejudicadas da qualidade de vida seriam explicadas pela insatisfação das puérperas com o lar, apoio recebido, condições sociais, vizinhos e dor (CONDELES PC, et al., 2019).

A presença do choro, suspiros, inquietação, irritabilidade, medo, sono perturbado, desconforto no baixo ventre e região lombo sacral, queixa de insegurança, desânimo, sofrimento espiritual, sentimento de desprezo, fome, dificuldade para relaxar, insatisfação com a situação e/ou ambiente, desconforto, prurido, calor ou frio e as expressões físicas de desconforto são indicadores do conforto prejudicado a serem avaliados pelo enfermeiro que assiste as parturientes e puérperas (GONÇALVES MCS, et al., 2020; RETT MT, et al., 2017).

As diretrizes nacionais recomendam à equipe de saúde que identifiquem e respeitem as crenças e valores das parturientes que influenciam a sua atitude em lidar com a dor durante o parto para lhes proporcionar alivio deste sintoma, por meio da analgesia regional, outras substâncias analgésicas e outras medidas não farmacológicas, como a aplicação de massagens e a imersão em água de banheira ou chuveiro (BRASIL, 2017).

Em algumas maternidades públicas de São Paulo - SP, os analgésicos, anti-inflamatórios e a crioterapia, com aplicação local de cubos de gelo em luva de látex, foram os principais métodos adotados para tratamento da dor perineal pós-parto normal e do edema perineal (PELECKIS MV, et al., 2017).

Para promover o conforto físico, deve-se investigar os fatores que predispõem e aliviam o desconforto, como roupa de cama com rugas e irritantes ambientais, reduzir e elimina-los, programar os cuidados de enfermagem para evitar interrupções desnecessárias e proporcionar períodos de descanso. Entre as medidas de promoção do conforto psicológico está a terapia de relaxamento, orientar a realização de respiração profunda, manter o ambiente com iluminação difusa e temperatura confortável quando possível (LIMA JVF, et al., 2016).

Além destes cuidados, as maternidades devem investir no acolhimento e nas práticas humanizadas para fortalecer o vínculo entre profissionais, usuárias, família e comunidade, bem como qualificar a atenção ao parto e puerpério (RETT MT, et al., 2017).

Também se faz necessário o referenciamento da puérpera para atenção básica, para garantir a continuidade dos cuidados de enfermagem para satisfazer suas necessidades de conforto, uma vez que 0 controle e alívio da dor proporcionará conforto físico e psicológico (LIMA JVF, et al., 2016).

A dor foi a dimensão da qualidade de vida mais prejudicada entre as mulheres que se auto declararam brancas, contrariando os resultados da Pesquisa Nacional sobre Parto e Nascimento, na qual as mulheres negras e pardas tiveram pré-natal e puerpério inadequados e maiores taxas de mortalidade materna. Esse desfecho releva as disparidades raciais no processo de atenção à gestação e ao parto que devem ser superadas e devem ser melhor exploradas por futuras investigações (LEAL MC, et al., 2017).

Também foi observada neste estudo a relação entre cesariana e baixo escore dos aspectos físicos, emocionais e mentais da qualidade de vida das puérperas que vivenciaram o NMM. De semelhante modo, as puérperas da Colômbia que vivenciaram o NMM também apresentaram a qualidade de vida prejudicada por consequências físicas, emocionais e psicológicas, as quais sofreram perdas da imagem corporal, autoestima, identidade e capacidade de produtividade econômica, além do sentimento de fracasso com relação ao sentimento de maternidade (VALENCIA-FLÓREZ B e AVIRIA-LONDOÑO MB, 2018). 
Em outros estudos, as puérperas que vivenciaram o NMM expressaram tanto gratidão pela vida salva como medo, tristeza, desesperança, desânimo e frustração referentes ao atendimento recebido na maternidade, também demonstraram algumas deficiências na atitude do pessoal de saúde, recursos físicos e atrasos no tratamento ansiedade decorrente a este evento traumático, além do sentimento de medo presente em seus cônjuges, sendo constatado que o fatalismo, as expectativas e a insegurança social contribuem significativamente para a vulnerabilidade das mesmas (TUNÇALP O, et al., 2012; MBALINDA SN, et al., 2015).

As mulheres atendidas em duas maternidades públicas de Aracaju - SE, que são referência para a gestação de médio e alto risco apresentaram associação entre NMM e sintomatologia de ansiedade e depressão, possivelmente devido ao baixo suporte social, antecedentes pessoais de transtornos de humor, histórico familiar de doença psiquiátrica, gravidez não planejada, baixo nível social e econômico das mesmas, além da precarização da assistência à saúde materna (SILVEIRA MS, et al., 2019).

Entre as puérperas assistidas por dois serviços de saúde de atenção à saúde da mulher de Ribeirão Preto$\mathrm{SP}$ e as que participaram de estudos realizados na África e Ásia a dispareunia prevaleceu entre as causas da qualidade de vida prejudicada após a ocorrência do NMM, fato este pouco explorado e que impede estas mulheres de terem orgasmos (ALVES LC, et al., 2020).

A paridade não foi preditora da qualidade de vida das puérperas que vivenciaram NMM deste estudo, tampouco da dor de puérperas assistidas em uma maternidade pública paranaense, entre as quais houve diferenças significativas para intensidade da dor perineal e incisão da cesariana (TOMASONI TA, et al., 2020).

A dor foi relatada pela maioria das puérperas que tiveram parto cesáreo em dois hospitais de São Paulo, especialmente na região suprapúbica e abdominal, as quais apresentaram dificuldades para sentar e andar. Já para as puérperas de um estudo no Recife-PE, a dor, as dificuldades para retomar as atividades habituais e o apoio de pessoas na recuperação após a cesárea foram decisivos para a qualidade de vida neste período (SANTOS JO, et al., 2016; MEDEIROS TML e MARCELINO JFQ, 2018).

A equipe de enfermagem deve estar capacitada para atender às puérperas com tais prejuízos na qualidade de vida, pois os cuidados de enfermagem ainda estão centrados no modelo biomédico, com a administração de medicações e orientações e medidas não farmacológicas para o alívio da dor (FIGUEIREDO JV, et al., 2018).

Como limitação deste estudo podemos destacar a dificuldade de encontrar outras pesquisas sobre qualidade de vida e NMM no período pós-operatório para ampliar as discussões dos dados que foram encontrados e está limitação também reforça a importância/relevância desta pesquisa. Como aplicação prática os dados por si só revelam/demostram um evento recorrente pouco explorado e que podem indicar para os serviços de saúde referência para essas mulheres a necessidade de melhoria na qualidade da atenção ao pré-natal e puerpério.

\section{CONCLUSÃO}

Neste estudo foi evidenciado que a qualidade de vida de mulheres que vivenciaram o near miss no período pós-parto foi prejudicada pela dor e limitações físicas e emocionais, as quais definem o diagnóstico de enfermagem de conforto prejudicado. Este achado deve embasar a atenção qualificada à saúde materna no período pós-natal, que além da prevenção e do tratamento das complicações e risco de morte, deve incluir a promoção da qualidade de vida das mulheres nessa fase tão vulnerável do ciclo reprodutivo.

\section{REFERÊNCIAS}

1. AGUIAR CA, TANAKA ACD. Memórias coletivas de mulheres que vivenciaram o near miss materno: necessidades de saúde e direitos humanos. Cad Saúde Pública, 2016; 32(9):e00161215.

2. ALVES LC, et al. Saúde sexual de mulheres seis meses após um evento de morbidade materna grave. Rev LatinoAm Enferm., 2020; 28:e3293.

3. ANGELINI ĆR, et al. Quality of life after an episode of severe maternal morbidity: evidence from a cohort study in Brazil. Biomed Research International, 2018; 1-10. 
4. ARANTES BM, et al. Fatores associados ao near miss materno em um hospital universitário. REFACS, 2020; 8(3).

5. BRASIL. MINISTÉRIO DA SAÚDE. SECRETARIA DE CIÊNCIA, TECNOLOGIA E INSUMOS ESTRATÉGICOS. DEPARTAMENTO DE GESTÃO E INCORPORACCÃO DE TECNOLOGIAS EM SAÚDE. Diretrizes nacionais de assistência ao parto normal: versão resumida. Brasília: Ministério da Saúde, 2017. 51p.

6. CABRAL FB, OLIVEIRA DLLC. Vulnerabilidade de puérperas na visão de equipes de saúde da família: ênfase em aspectos geracionais e adolescência. Rev Esc Enferm USP, 2010; 44(2): 368-375.

7. CICONELLI RM. Tradução para o português e validação do questionário genérico de avaliação de qualidade de vida Medical Outcomes Study 36-Item Short-form Health Survey (SF-36) [tese]. São Paulo: Universidade Federal de São Paulo; 1997

8. CHICAS AAN, et al. Mortalidad y morbilidad materna externa, Hospital Escuela Universitario 2015: estudio casocontrol. Rev Méd Hondur., 2018; 86(3/4):96-101.

9. CONDELES PC, et al. Qualidade de vida no período puerperal: importância e satisfação. Rev Rene, 2019; 20: e41421.

10. DIAS MAB, et al. Incidência do Near Miss Materno no Parto e Pós-parto hospitalar: dados da pesquisa Nascer no Brasil. Cad Saúde Pública, 2014; 30(Supl 1):S1-12.

11. FERREIRA EC, et al. Saúde geral e reprodutiva entre mulheres após um episódio de morbidade materna grave: resultados do estudo COMMAG. Int J Gynecol Obstet., 2020; 150:83-91.

12. FIGUEIREDO JV, et al. Pain in the immediate puerperium: nursing care contribution. Rev Bras Enferm., 2018; 71(Suppl 3):1343-50.

13. GONÇALVES MCS, et al. Validação das características definidoras do diagnóstico de enfermagem conforto prejudicado em oncologia. Acta Paul Enferm.,2016; 29(1):115-24.

14. IWUH IA, FAWCUS S, SCHOEMAN L. Maternal near-miss audit in the Metro West maternity service, Cape Town, South Africa: a retrospective observational study. S Afr Med J., 2018; 108(3):171-5.

15. LEAL MC, et al. A cor da dor: iniquidades raciais na atenção pré-natal e ao parto no Brasil. Cad Saúde Pública, 2017; 33:e00078816

16. LIMA JVF, et al. Utilidade da teoria do conforto para o cuidado clínico de enfermagem à puérpera: análise crítica. Rev Gaúcha Enferm., 2016; 37(4):e65022.

17. LONGHI SAT, PETERLINI OLG. Comissão interna de near miss materno em um hospital da Rede Mãe Paranaense no sudoeste do Paraná: implantação, desafios e perspectivas Rev Saúde Públ Paraná, 2019; 2(Suppl 1):21-30.

18. MBALINDA SN, et al. Male partners' perceptions of maternal near miss obstetric morbidity experienced by their spouses. Reprod Health, 2015; 12(1):23.

19. MEDEIROS TML, MARCELINO JFQ. Percepção de puérperas sobre o seu desempenho ocupacional no pósoperatório da cesariana. Cad Bras Ter Ocup., 2018; 26(1):97-109.

20. MEKANGO DE, et al. Determinants of maternal near miss among women in public hospital maternity wards in Northern Ethiopia: a facility based case-control study. Plos Oone, 2017; 12(9):e0183886.

21. ORGANIZAÇÃO MUNDIAL DA SAÚDE (OM). Avaliação da qualidade do cuidado nas complicações graves da gestação: a abordagem do near miss da OMS para a saúde materna. Geneva: OMS, 2011. 34 p.

22. ORGÁNIZAÇÃO MUNDIAL DE SAÚDE (OMS). Mulheres e saúde: evidências de hoje, agenda de amanhã. 2011. Disponível em: https://www.who.int/eportuguese/publications/Mulheres_Saude.pdf?ua=1. Acessado em: 09 nov. 2020.

23. OKUN ML, et al. Poor sleep quality increases symptoms of depression and anxiety in postpartum women. J Behav Med, 2018; 41:703-10.

24. PARANÁ. Secretaria de Estado de Saúde do Paraná. Superintendência de Atenção à Saúde. Linha Guia da Rede Mãe Paranaense, 2018.

25. PELECKIS MV, et al. Terapias de alívio da dor perineal após o pós-parto. Texto Contexto Enferm., 2017; 26(2):e05880015.

26. PINHEIRO DLFL, et al. Gestational Outcomes in Patients with Severe Maternal Morbidity Caused by Hypertensive Syndromes. Rev Bras Ginecol Obstet., 2020; 42(2):74-80.

27. RETT MT, et al. Satisfação e percepção de dor em puérperas: um estudo comparativo após parto vaginal e cesariana em maternidades públicas de Aracaju. ABCS Health Sciences, 2017; 42(2).

28. REZENDE FILHO J e MONTENEGRO CAB. Obstetrícia fundamental. Guanabara Koogan; Rio de Janeiro, 2014; (11): 106-107.

29. SANTANA DS, et al., Near miss materno: entendendo e aplicando o conceito. Rev Med (São Paulo), 2018; 97(2):18794.

30. SANTOS JO, et al. Evaluation of pain during postpartum: study comparing types of birth. J Health Sci Inst., 2016; 34(4):200-5.

31. SILVA SGF, et al. Influence of sociodemographic, clinical, obstetric and neonatal variables on postpartum quality of life. Rev Enferm UERJ, 2019; 27:e44636.

32. SILVEIRA MS, et al. Ansiedade e depressão na morbidade materna grave e near miss. Psicologia: teoria e pesquisa, 2019; 35:e35442.

33. SOMA-PILLAY P, MAKIN JD, PATTINSON RC. Qualidade de vida um ano após um evento de near miss materno. Int J Gynecol Obstet., 2018; 141:133-8.

34. SULTANA S, et al. Clinical spectrum of near-miss cases in obstetrics. Cureus, 2019; 11(5):e4641.

35. TOMASONI TA, et al. Intensidade de dor e desconfortos puerperais imediatos. BrJP, 2020; 3(3):217-221.

36. TUNÇALP O, et al. Listening to women's voices: the quality of care of women experiencing severe maternal morbidity, in Accra, Ghana. PLos One, 2012; 7(8):1-8.

37. VALENCIA-FLÓREZ B e GAVIRIA-LONDOÑO MB. Vida vivida: experiencias de morbilidad materna extrema. Relatos de mujeres sobrevivientes y cuidadoras. Subregión del Urabá antioqueño, 2016. Rev Fac Nac Salud Pública, 2018; 36(3).

38. VIDAL CEL, et al. Morbidade materna grave na microrregião de Barbacena/MG. Cad Saúde Colet., 2016; 24(2): 1318.

39. VIEIRA SN, et al. Evaluation of nursing care in post-partum hemorrhaging. J Nurs UFPE, 2018; 12(12):3247-53.

40. WORLD HEALTH ORGANIZATION (WHO). Health statistics and health information systems. Maternal mortality ratio (per 100000 live births). Disponível em: http://www.who.int/healthinfo/statistics/indmaternalmortality/en/. Acessado em: 09 nov. 2020. 